\title{
Trajectory, orbit, and spectroscopic analysis of a bright fireball observed over Spain on April 13, 2013
}

\author{
José M. Madiedo ${ }^{1,2}$, Josep M. Trigo-Rodríguez ${ }^{3}$, Jaime Zamorano ${ }^{4}$, Leonor Ana-Hernández ${ }^{5}$, Jaime Izquierdo ${ }^{4}$, \\ José L. Ortiz ${ }^{6}$, Aberto J. Castro-Tirado ${ }^{6}$, Alejandro Sánchez de Miguel ${ }^{4}$, Francisco Ocaña ${ }^{4}$, Sensi Pastor ${ }^{7}$, \\ José A. de los Reyes ${ }^{7}$, David Galadî ${ }^{8}$, Enrique de Guindos ${ }^{8}$, Faustino Organero ${ }^{5}$, \\ Fernando Fonseca ${ }^{5}$, and Jesús Cabrera-Caño ${ }^{1}$

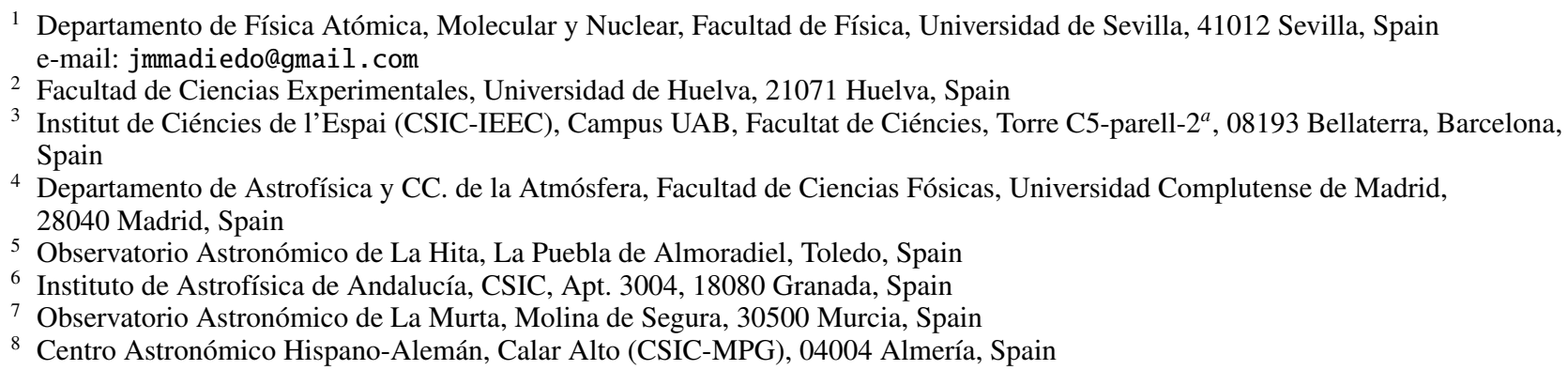

Received 22 June 2013 / Accepted 7 August 2014

\section{ABSTRACT}

\begin{abstract}
On April 13, 2013 a very bright fireball with an absolute magnitude of $-13.0 \pm 0.5$ was recorded over the center of Spain. This sporadic event, which was witnessed by numerous casual observers throughout the whole country, was imaged from seven meteorobserving stations operated by the Spanish Meteor Network (SPMN), and its emission spectrum was also obtained in the framework of our meteor spectroscopy campaign. The atmospheric trajectory of the bolide and the heliocentric orbit of the parent meteoroid are analyzed here. The spectrum reveals a chondritic nature for this particle, which was following a Jupiter family comet orbit before its encounter with the Earth. In addition, the emission spectrum of the meteoric afterglow was recorded during about $0.8 \mathrm{~s}$. The main emission lines appearing in this signal were identified and their evolution with time is also discussed. Afterglow spectra are not abundant in the literature, and these can provide important clues about the physical proceses taking place in meteoric persistent trains.
\end{abstract}

Key words. meteorites, meteors, meteoroids

\section{Introduction}

The analysis of fireball events provides information about the behavior of large cometary and asteroidal meteoroids in the Earth's atmosphere. The detailed recordings performed from meteorobserving stations allows determining the orbit of these particles, but also their mass and strength. Milley et al. (2007) found that the light produced during the ablation of meteoroids in the atmosphere is a combination of either continuum or unresolved spectral line emission, along with spectral emission lines of neutral and single-ionized species such as $\mathrm{Ca}, \mathrm{Fe}, \mathrm{Na}, \mathrm{N}, \mathrm{Mg}, \mathrm{Si}$, and $\mathrm{O}$. But emission bands of different molecules (such as $\mathrm{CN}, \mathrm{N}_{2}$, $\mathrm{C}_{2}, \mathrm{FeO}$, and $\mathrm{CaO}$ ), have also been observed (Borovička 1994; Jenniskens 2004). Spectroscopy clearly also plays a fundamental role in the analysis of these events, since fireball emission spectra can provide very useful data about the physicochemical conditions in meteor plasmas and about the composition of the progenitor meteoroids (see, e.g., Borovička 1993; 2005; Babadzhanov \& Kokhirova 2007; Jenniskens 2007). In addition, these compositions can be employed to infer key information about the parent bodies of these particles (Koten et al. 2006; Madiedo et al. 2013a, 2014).

In the evening of April 13, 2013, a fireball with an absolute magnitude of $-13.0 \pm 0.5$ flew over the center of Spain. Because of its extraordinary luminosity and favorable weather conditions on most of the Iberian Peninsula, it was witnessed by numerous casual observers throughout the country. No thunderlike or electrophonic sounds were reported. This sporadic bolide was confirmed by our meteor-observing stations, which recorded the fireball at $21 \mathrm{~h} 43 \mathrm{~m} 55.4 \pm 0.1 \mathrm{~s}$ UTC (about $16 \mathrm{~min}$ before local midnight). The event was included in our fireball database with the Spanish Meteor Network (SPMN) code 130413, which was assigned after the observing date with format yymmdd. We named this extraordinary fireball Serranillos del Valle, since its maximum brightness was reached when the bolide was located next to the zenith of this town, at about $26 \mathrm{~km}$ southwest of Madrid. Here we analyze the atmospheric trajectory and radiant of the Serranillos del Valle bolide. The heliocentric orbit of the parent meteoroid is also obtained. In addition, we discuss the emission spectrum produced during the ablation of this particle in the atmosphere In this way, we have derived information about the bulk chemistry of this particle. The afterglow spectrum, which was recorded during about $0.8 \mathrm{~s}$ after the main flare took place, is also discussed.

\section{Instrumentation and methods}

The SPMN meteor-observing stations that recorded the event discussed here (Table 1) employ an array of low-lux monochrome 8-bit CCD cameras (models 902H2 and 902H 
Table 1. Geographical coordinates of the meteor-observing stations that imaged the fireball discussed in this work.

\begin{tabular}{lcccc}
\hline \hline Station \# & Station name & Latitude $(\mathrm{N})$ & Longitude $(\mathrm{W})$ & Alt. (m) \\
\hline 1 & Sevilla & $05^{\circ} 58^{\prime} 50^{\prime \prime}$ & $37^{\circ} 20^{\prime} 46^{\prime \prime}$ & 28 \\
2 & La Hita & $03^{\circ} 11^{\prime} 00^{\prime \prime}$ & $39^{\circ} 34^{\prime} 06^{\prime \prime}$ & 674 \\
3 & Huelva & $06^{\circ} 56^{\prime} 11^{\prime \prime}$ & $37^{\circ} 15^{\prime} 10^{\prime \prime}$ & 25 \\
4 & El Arenosillo & $06^{\circ} 43^{\prime} 58^{\prime \prime}$ & $37^{\circ} 06^{\prime} 16^{\prime \prime}$ & 40 \\
5 & Villaverde del Ducado & $02^{\circ} 29^{\prime} 29^{\prime \prime}$ & $41^{\circ} 00^{\prime} 04^{\prime \prime}$ & 1100 \\
6 & Madrid (UCM) & $03^{\circ} 43^{\prime} 34^{\prime \prime}$ & $40^{\circ} 27^{\prime} 03^{\prime \prime}$ & 640 \\
7 & Molina de Segura & $01^{\circ} 09^{\prime} 50^{\prime \prime}$ & $38^{\circ} 05^{\prime} 54^{\prime \prime}$ & 60 \\
\hline
\end{tabular}

Ultimate, from Watec Corporation) that operate, weather permitting, during the whole night. Station \#1 also employs several color CCD video cameras. Although these were set up to monitor the sky during the day, we usually keep them working also during the night to image events with brightness higher than that corresponding to an apparent magnitude of about -4 . Our monochrome and color devices, whose operation is detailed elsewhere (Madiedo \& Trigo-Rodríguez 2008; Madiedo et al. 2010; Madiedo 2014), generate interlaced video sequences with a frame rate of $25 \mathrm{fps}$ and a resolution of $720 \times 576$ pixels. The fireball was also recorded by a low-resolution video camera operating at the Calar Alto Astronomical Observatory. In addition, it was captured by a digital photographic camera (model 10D, manufactured by Canon Inc.) endowed with an $8 \mathrm{~mm}$ fisheye lens, which was taking images in the framework of an education and outreach activity developed at La Hita Astronomical Observatory. In addition, the emission spectrum produced during the ablation of the parent meteoroid in the atmosphere was recorded from several SPMN meteor-observing stations. Thus, we perform a systematic spectroscopic monitoring since 2006 to infer information about the chemical composition of meteoroids. For this purpose we employ holographic diffraction gratings (500 or 1000 lines/mm, depending on the device) attached to the objective lens of our low-lux Watec cameras (Trigo-Rodriguez et al. 2009; Madiedo et al. 2013b-d). These video spectrographs can obtain the spectrum of meteor events with brightness higher than that corresponding to an apparent magnitude of around -4 . The first step of the data reduction involved deinterlacing the video sequences by separating the even and odd fields contained in each video frame. Then, these frames were processed by hand to measure the plate $(x, y)$ coordinates of the fireball along its apparent trajectory in the night sky from each observing meteor station. The planes-intersection method (Ceplecha 1987) was employed to derive the atmospheric trajectory and radiant of the event. This calculation was performed with our AMALTHEA software by following the procedure described in Madiedo et al. (2013b, 2013d). When these data were known, the software computed the heliocentric orbit of the parent meteoroid by means of the procedure described in Ceplecha (1987).

\section{Observations: atmospheric path, radiant, and orbit}

Figure 1a shows an instant image of the Serranillos del Valle fireball as seen from La Hita Astronomical Observatory during its maximum brightness. The persistent train recorded immediately after the end of the bolide is shown in Fig. 1b. The significant increase in the luminosity of the environment as a consequence of the fireball is obvious. Image 1c was taken by a surveillance camera at the Calar Alto Astronomical Observatory (located at about $330 \mathrm{~km}$ from the epicenter of the event), which generates video imagery at a rate of one frame per second. Thus, because of the relatively low temporal precision, we cannot be sure if the frame shown in that figure corresponds exactly to the maximum brightness phase of the event. A composite image taken from our meteor station at Molina de Segura is shown in Fig. 1d. The long-exposure (30 s) image taken by the digital camera operating at La Hita Astronomical Observatory is shown in Fig. 2. The reports sent by the casual witnesses did not provide precise positional data, but all of them coincided in the extraordinary brightness of the bolide. In particular, those located in the center of Spain, near the epicenter of the event, remarked that the night turned into day for a fraction of a second. They also reported a greenish to bluish color for the fireball and that it was relatively slow, lasting for several seconds.

According to the calculated atmospheric trajectory, the bolide began at $101.4 \pm 0.5 \mathrm{~km}$ above the ground level, and the preatmospheric velocity of the meteoroid was $V_{\infty}=19.1 \pm$ $0.5 \mathrm{~km} \mathrm{~s}^{-1}$. The apparent radiant was located at the coordinates $\alpha=167.78 \pm 0.02^{\circ}, \delta=-8.08 \pm 0.02^{\circ}$ and the atmospheric path had an inclination of around $42^{\circ}$ with respect to the ground. When the fireball penetrated the atmosphere to a height of $86.5 \pm 0.5 \mathrm{~km}$ it exhibited a very bright flare, reaching then its maximum brightness. The photometric analysis of the images revealed that this maximum luminosity corresponded to an absolute magnitude of $-13.0 \pm 0.5$. The bolide continued to penetrate the atmosphere to a final height of $68.1 \pm 0.5 \mathrm{~km}$. Table 2 contains the main parameters characterizing this atmospheric path. The projection on the ecliptic plane of the heliocentric orbit of the meteoroid is plotted in Fig. 1e, and the corresponding orbital parameters are also summarized in Table 2.

\section{Results}

\subsection{Photometric analysis}

The light curve of the bolide (Fig. 3), which lasted about $3.1 \mathrm{~s}$, was obtained from the photometric analysis of the video sequences obtained by our CCD cameras. The peaks in this curve correspond to the main flares exhibited by the fireball. These took place at times $t_{1}=1.9, t_{2}=2.2$, and $t_{3}=2.8 \mathrm{~s}$, respectively, after the beginning of the event, that is, during the second half of its atmospheric trajectory. The most intense of these flares was the first one, which took place at a height of $86.5 \pm 0.5 \mathrm{~km}$ above the ground level. The second of these followed almost immediately and, in fact, was not reported by the casual observers since it was almost completely masked by the first one. After the third flare, the surviving material continued to ablate to a final height of $68.1 \mathrm{~km}$. 


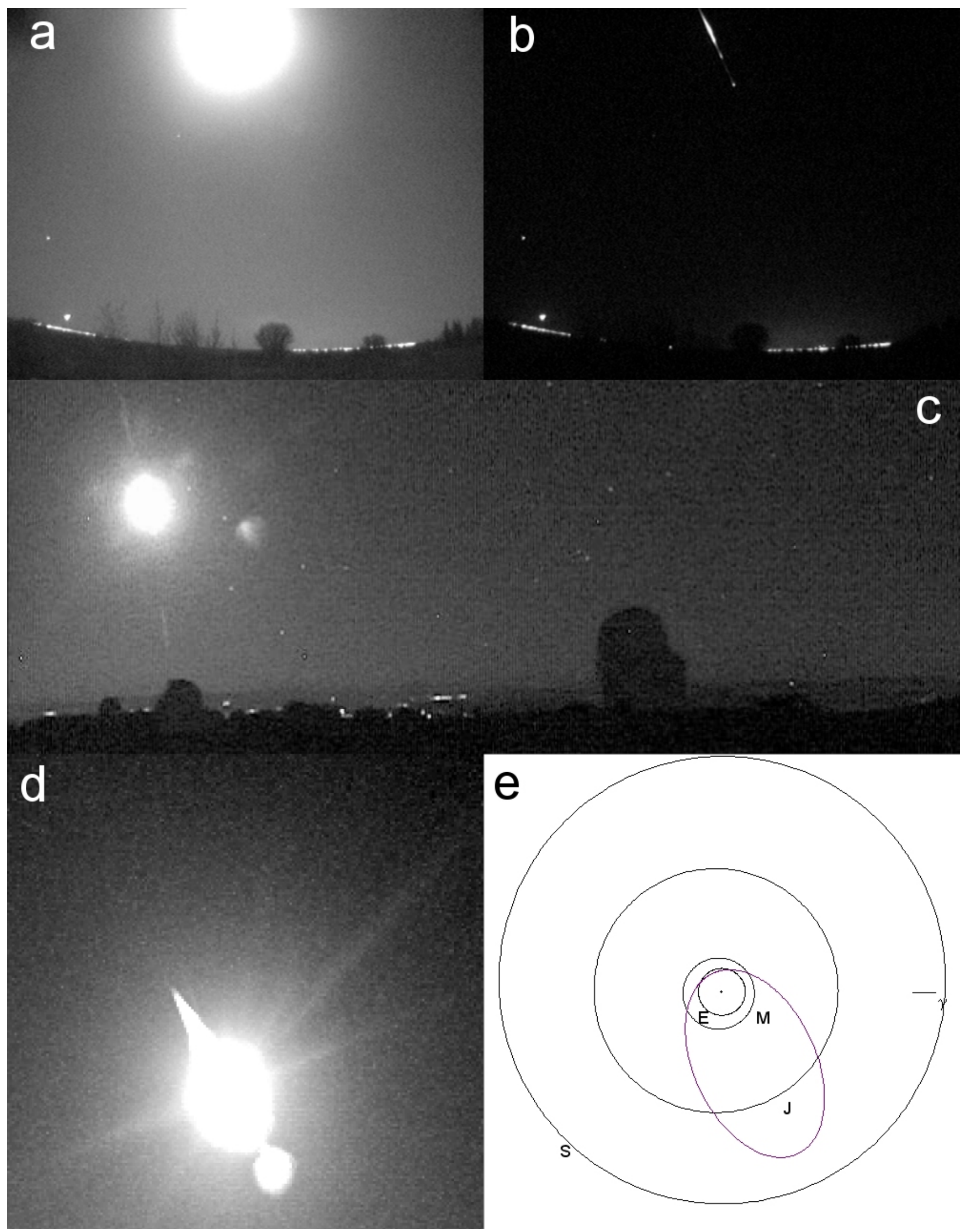

Fig. 1. Video frames showing the brightest phase a) and afterglow b) of the fireball as imaged from La Hita Astronomical Observatory. c) Image taken from Calar Alto during the brightest phase of the fireball. d) Composite image of the event taken from Molina de Segura. e) Projection on the ecliptic plane of the heliocentric orbit of the meteoroid. 


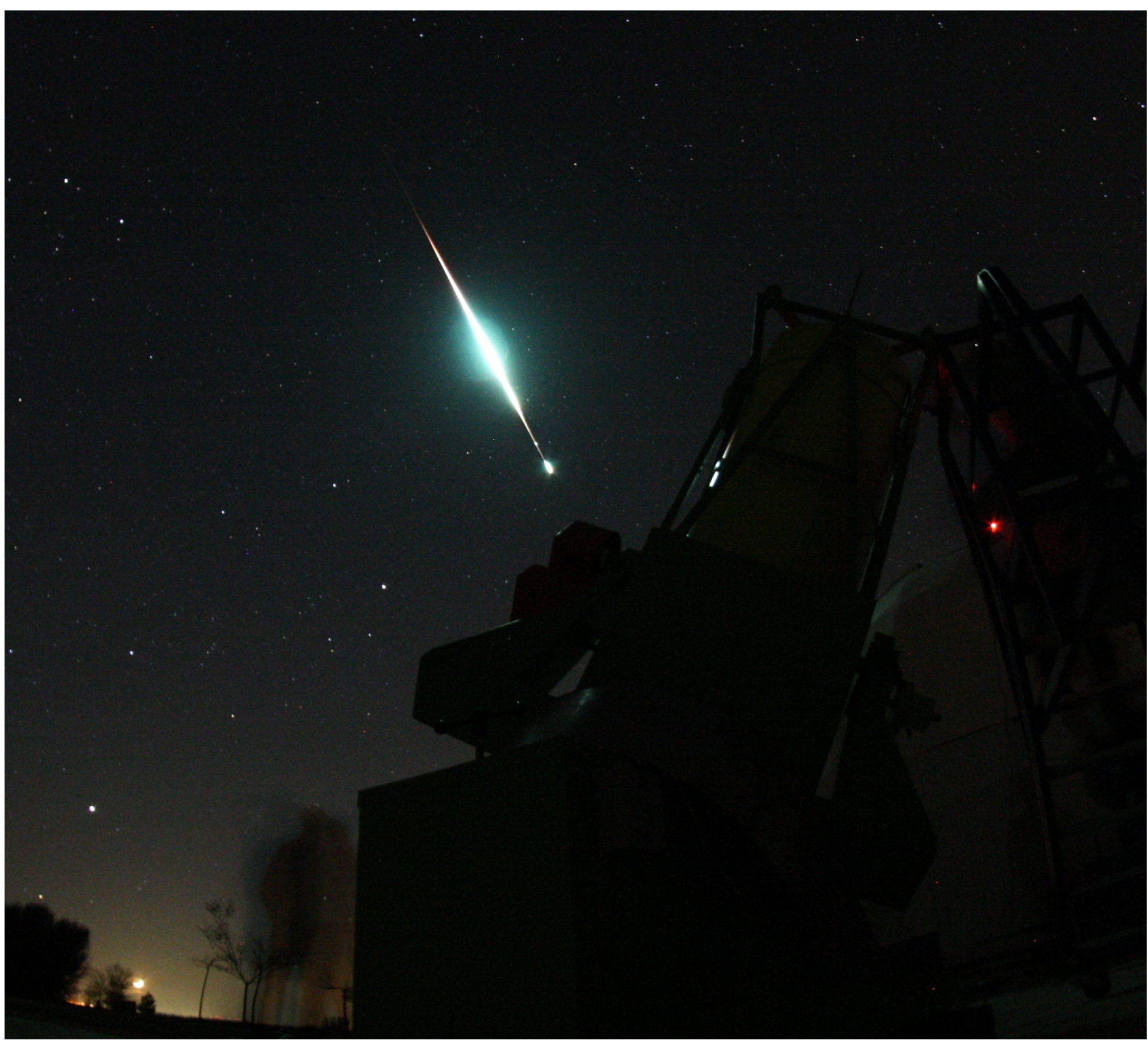

Fig. 2. Photograph taken by Leonor Ana-Hernández of the SPMN130413 fireball from La Hita Astronomical Observatory.

Table 2. Trajectory, radiant and orbital data obtained for the SPMN130413 fireball (J2000).

\begin{tabular}{cccccccccccc}
\hline \hline $\begin{array}{c}H_{\mathrm{b}} \\
(\mathrm{km})\end{array}$ & $\begin{array}{c}H_{\mathrm{e}} \\
(\mathrm{km})\end{array}$ & $\begin{array}{c}\alpha_{\mathrm{g}} \\
\left({ }^{\circ}\right)\end{array}$ & $\begin{array}{c}\delta_{\mathrm{g}} \\
\left({ }^{\circ}\right)\end{array}$ & $\begin{array}{c}V_{\infty} \\
\left(\mathrm{km} \mathrm{s}^{-1}\right)\end{array}$ & $\begin{array}{c}V_{\mathrm{g}} \\
\left(\mathrm{km} \mathrm{s}^{-1}\right)\end{array}$ & $\begin{array}{c}V_{\mathrm{h}} \\
\left(\mathrm{km} \mathrm{s}^{-1}\right)\end{array}$ & $\begin{array}{c}a \\
(\mathrm{AU})\end{array}$ & $e$ & $\begin{array}{c}i \\
\left({ }^{\circ}\right)\end{array}$ & $\begin{array}{c}\Omega \\
\left({ }^{\circ}\right)\end{array}$ & $\begin{array}{c}\omega \\
\left({ }^{\circ}\right)\end{array}$ \\
\hline 101.4 & 68.1 & 166.69 & -13.2 & 19.1 & 15.5 & 39.5 & 4.3 & 0.79 & 7.1 & 203.9450 & 40.95 \\
\pm 0.5 & \pm 0.5 & \pm 0.03 & \pm 0.2 & \pm 0.5 & \pm 0.5 & \pm 0.5 & \pm 0.7 & \pm 0.04 & \pm 0.1 & $\pm 10^{-4}$ & \pm 0.03 \\
\hline
\end{tabular}

The initial mass $m_{\infty}$ of the meteoroid was inferred from this light curve by employing the classical meteor luminous equation:

$m_{\infty}=2 \int_{t_{\mathrm{b}}}^{t_{\mathrm{e}}} \frac{I}{\tau v^{2}} \mathrm{~d} t$

In this relationship $t_{\mathrm{b}}$ and $t_{\mathrm{e}}$ are the instants corresponding to the beginning and the end of the luminous trajectory of the event, with $I$ being the measured luminosity of the fireball. This luminosity is expressed as a function of the absolute magnitude $M$ by means of the equation

$I=10^{-0.4 M}$.
The velocity-dependent luminous efficiency $\tau$ was calculated from the relationships given by Ceplecha \& McCrosky (1976). Thus, the inferred mass of the meteoroid is $40 \pm 5 \mathrm{~kg}$.

\subsection{Tensile strength}

We estimated the strength of the meteoroid by calculating the aerodynamic pressure under which the flares took place. When this pressure equates the mechanical strength of the meteoroid, the particle breaks up. This produces a quick release of dust grains and an increase in the effective cross-section of the 
Table 3. Aerodynamic pressure for the main flares exhibited by the fireball.

\begin{tabular}{lccc}
\hline \hline Flare \# & Height $(\mathrm{km})$ & Velocity $\left(\mathrm{km} \mathrm{s}^{-1}\right)$ & Aerodynamic pressure $(\mathrm{Pa})$ \\
\hline 1 & $86.5 \pm 0.5$ & $18.8 \pm 0.5$ & $(1.8 \pm 0.3) \times 10^{3}$ \\
2 & $81.8 \pm 0.5$ & $18.7 \pm 0.5$ & $(4.1 \pm 0.3) \times 10^{3}$ \\
3 & $70.2 \pm 0.5$ & $17.9 \pm 0.5$ & $(2.3 \pm 0.3) \times 10^{4}$ \\
\hline
\end{tabular}

Notes. The position of these break-up events has been highlighted in the light curve shown in Fig. 3.

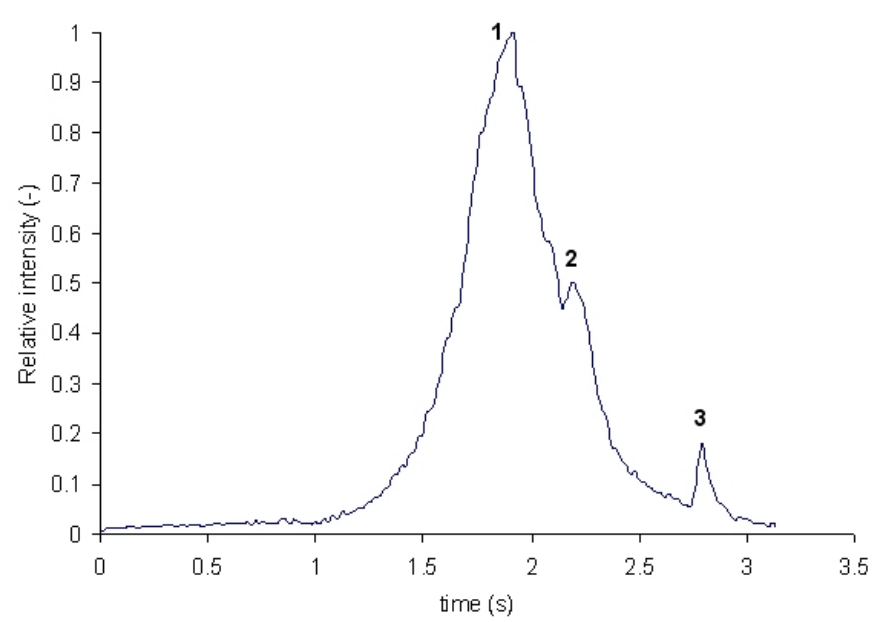

Fig. 3. Light curve (relative pixel intensity vs. time) of the SPMN130413 fireball.

meteor, which gives rise in turn to a sudden increase in luminosity. (Trigo-Rodriguez \& Llorca 2006, 2007). According to this approach, the tensile strength $S$ was obtained by means of the following equation (Bronshten 1981):

$S=\rho_{\mathrm{atm}} v^{2}$,

where $v$ and $\rho_{\text {atm }}$ are the velocity of the meteoroid and the atmospheric density at the height where the flare takes place. We calculated this density by following the U.S. standard atmosphere model (U.S. Standard Atmosphere 1976). The values obtained for the three flares highlighted in Fig. 2 are listed in Table 3, together with the corresponding heights and velocities.

\subsection{Fireball spectrum}

The emission spectrum of the fireball was obtained by three video spectrographs operating at observing stations 4,5 and 6 . These slitless devices are sensitive in the range between 310 and $950 \mathrm{~nm}$, approximately. During the main flare, most of the emission lines in the spectrum were saturated, and therefore the signal obtained at that stage was of limited use. To analyze this spectrum we employed our CHIMET software, which calibrated the signal in wavelengths by identifying typical lines that appear in meteor spectra (Madiedo et al. 2011). Thus, according to multiplet numbers given by Moore (1945), this calibration was performed by means of the $\mathrm{Na} \mathrm{I}-1, \mathrm{Mg} \mathrm{I}-2, \mathrm{Fe} \mathrm{I}-41$, Ca I-2, and Fe I-15 multiplets. Then, the signal was corrected by taking into account the spectral sensitivity of the recording device. The calibrated spectrum corresponding to the instant immediately before that flare, when the fireball was located at a height of about $87 \mathrm{~km}$ above the ground level, is plotted in Fig. 4. The main lines identified in the signal are highlighted. The strongest

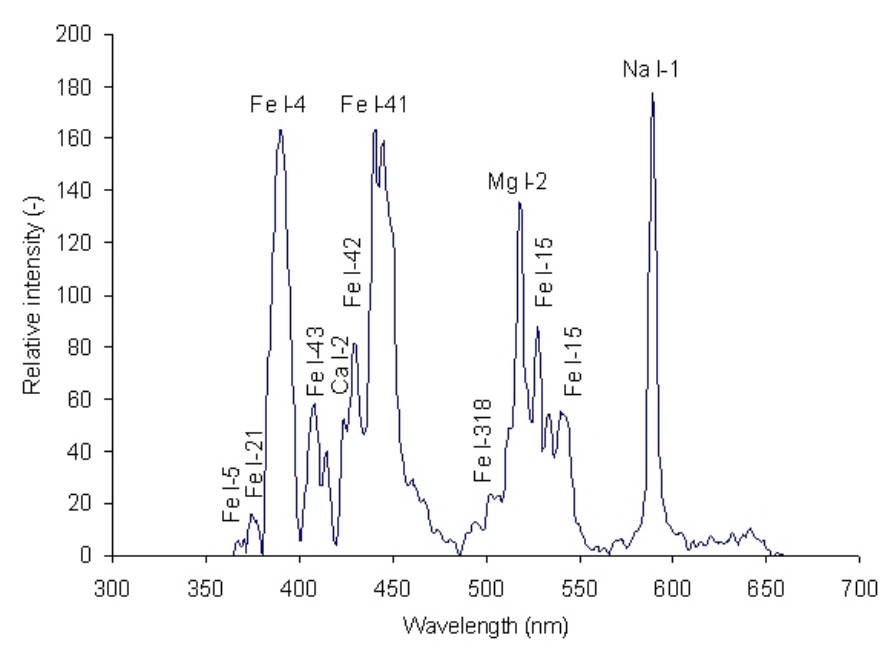

Fig. 4. Calibrated emission spectrum of the SPMN130413 bolide.

emission corresponds to the Na I-1 doublet at $588.9 \mathrm{~nm}$, followed by several Fe I multiplets (mainly the Fe I-41 line at $441.5 \mathrm{~nm}$ and the Fe I-4 multiplet at $389.9 \mathrm{~nm}$ ). The contribution from the $\mathrm{Mg} \mathrm{I}-2$ triplet $(517.2 \mathrm{~nm})$ is also very noticeable.

\subsection{Afterglow spectrum}

A persistent train is often formed at the position of a bright meteor flare, and the brightness of these trains, which can exceed magnitude -5 , decreases quickly during the so-called afterglow phase (Borovicka 2006). Thus, as a consequence of the brightest flare exhibited by this fireball, a persistent train (Fig. 1b) was observed with the naked eye by casual witnesses during several minutes. During the first $0.8 \mathrm{~s}$ after this flare took place, the afterglow was bright enough to produce an emission spectrum that was recorded by one of our video spectrographs. Figure 5 shows the afterglow spectrum recorded $0.4 \mathrm{~s}$ after the flare took place. Afterglow spectra can provide useful information about physical processes that occur in persistent meteor trains. However, such spectra are not abundant in the literature (Borovička \& Jenniskens 2000; Jenniskens et al. 2000; Abe et al. 2005).

The afterglow spectrum of the SPMN130413 bolide was also calibrated in wavelengths by following the same procedure as employed for the previously discussed fireball spectrum. In the signal we identified the emission from $\mathrm{Na} \mathrm{I}-1, \mathrm{Mg} \mathrm{I}-2, \mathrm{Ca} \mathrm{I}-2$ and several Fe I multiplets. Multiplets indicated in Fig. 5 are well above the noise level of the images.

\section{Discussion}

The radiant and orbital data (Table 2) confirm the sporadic nature of this event, since they do not fit any known meteoroid stream and the radiant is well outside the antihelion source 


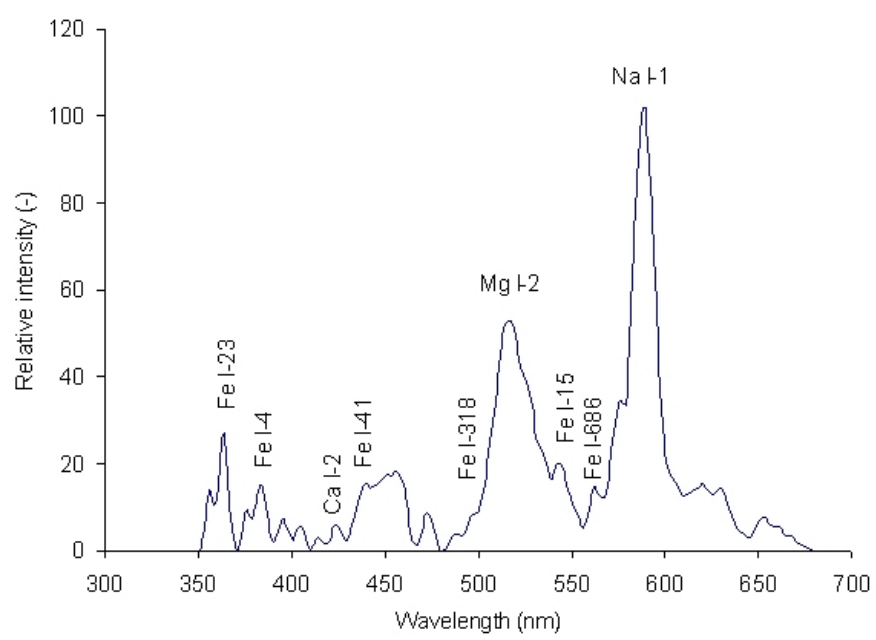

Fig. 5. Calibrated afterglow spectrum at $t=0.4 \mathrm{~s}$ after the main flare exhibited by the SPMN130413 bolide.

region. The Tisserand parameter with respect to Jupiter yields $T_{\mathrm{J}}=2.3 \pm 0.2$ and the period of revolution of the meteoroid was $P=8.9 \pm 1.7$ years. This means that this particle was following a Jupiter family comet (JFC) orbit before impacting our planet. To find the potential parent comet of the meteoroid we employed the Orbital Association Software (ORAS), which performs a search among the objects listed in the NeoDys ${ }^{1}$ and Minor Planet Center $^{2}$ databases. This software, which was developed by the first author and is described in detail in Madiedo et al. (2013b), can employ the dissimilarity criteria proposed by Southworth \& Hawkins (1963), Drummond (1981), Jopek (1993), Valsecchi et al. (1999), and Jenniskens (2008). No parent body was found among the objects currently included in these databases, however, which means that the Serranillos del Valle meteoroid might be part of a relatively high-inclination JFC that has not yet been discovered. With a mass of $40 \pm 5 \mathrm{~kg}$, the diameter of this meteoroid yields $31 \pm 1 \mathrm{~cm}$ for a bulk density of $2.4 \mathrm{~g} \mathrm{~cm}^{-3}$. For a bulk density of $3.7 \mathrm{~g} \mathrm{~cm}^{-3}$ this size would be $27 \pm 1 \mathrm{~cm}$. However, according to the empirical end height criterion proposed by Ceplecha \& McCrosky (1976), the fireball would be classified as type IIIB. These events are produced by the most fragile and weakest cometary meteoroids, which have an average bulk density of about $0.3 \mathrm{~g} \mathrm{~cm}^{-3}$. According to this, the diameter of the Serranillos del Valle meteoroid would be $63 \pm 3 \mathrm{~cm}$.

According to the values listed in Table 3 this, the brightest phase of the fireball (which corresponded to the first flare) took place under an aerodynamic pressure of $\left(1.8 \pm 0.3 \times 10^{3} \mathrm{~Pa}\right.$. This value is about one order of magnitude lower than that corresponding to the average tensile strength determined for sporadic meteoroids and one order of magnitude higher than the strength exhibited by meteoroids belonging to the October Draconid stream (Trigo-Rodríguez \& Llorca 2006, 2007). In any event, the value is consistent with the cometary nature of the impactor.

We investigated the nature of the meteoroid by analyzing the relative intensity of the $\mathrm{Na} \mathrm{I}-1, \mathrm{Mg} \mathrm{I}-2$, and Fe I-15 multiplets in the fireball spectrum (Borovička et al. 2005). For this purpose, the intensity of the corresponding emission lines was measured frame by frame in the video that contains the spectrum and then

\footnotetext{
1 http://newton.dm.unipi.it/neodys/

2 http://wwW . minorplanetcenter.org/iau/mpc.html
}

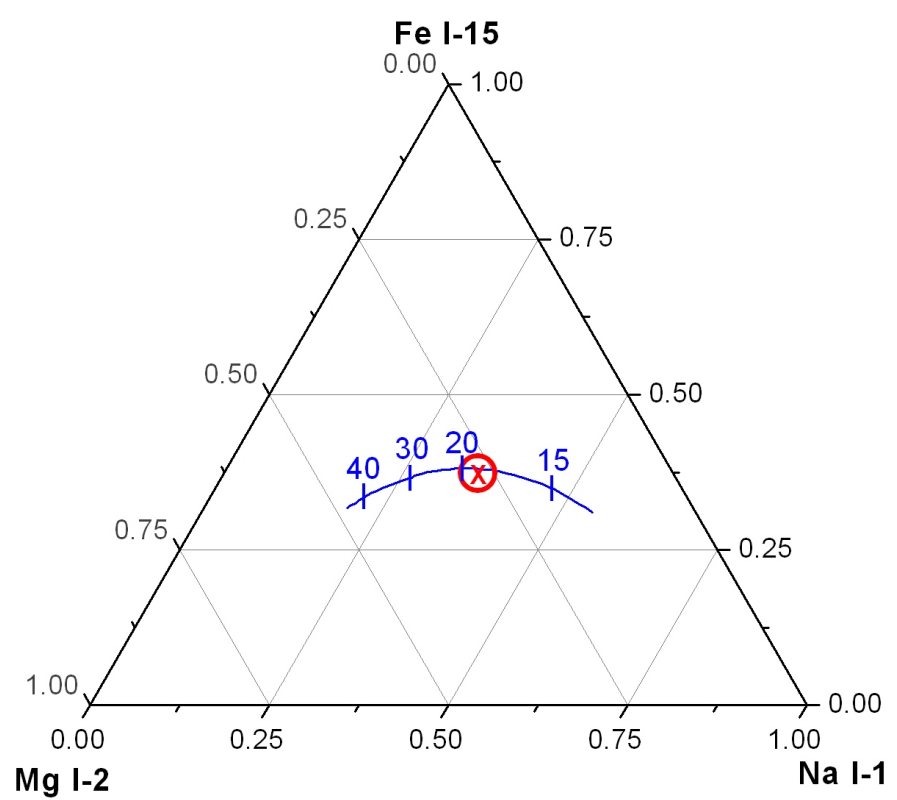

Fig. 6. Solid line: expected relative intensity, as a function of meteor velocity in $\mathrm{km} \mathrm{s}^{-1}$, of the Mg I-2, Na I-1 and Fe I-15 multiplets for chondritic meteoroids (Borovička et al. 2005). Cross: experimental relative intensity obtained from the spectrum of the "Serranillos del Valle" fireball. The circle corresponds to the uncertainty in the determination of this point.

corrected for the instrumental efficiency. Frames where the spectrum was saturated because of the high luminosity of the fireball were ignored. The contributions in each unsaturated frame were then added to obtain the integrated intensity for each line along the meteor path. In this way, we obtained a $\mathrm{Na} / \mathrm{Mg}$ intensity ratio of $1.32 \pm 0.09$ and a Fe/Mg ratio of $1.53 \pm 0.11$. The $\mathrm{Na} / \mathrm{Mg}$ intensity ratio fits the result expected for meteoroids with chondritic composition fairly well when the meteor velocity is of about $19 \mathrm{~km} \mathrm{~s}^{-1}$ (Fig. 5 in Borovička et al. 2005). We have plotted the $\mathrm{Na} \mathrm{I}-1, \mathrm{Mg} \mathrm{I}-2$, and Fe I-15 relative intensities in a ternary diagram (Fig. 6). The solid curve in this diagram corresponds to the expected relative intensity, as a function of meteor velocity, for chondritic meteoroids (Borovička et al. 2005). This plot reveals that, according to the classification given by Borovička et al. (2005), the emission spectrum of the Serranillos del Valle fireball can be considered normal. Thus, the position of the point that describes the fireball spectrum in this ternary diagram confirms the chondritic nature of the parent meteoroid. In fact, this point agrees very well with the expected relative intensity for a meteor velocity of about $19 \mathrm{~km} \mathrm{~s}^{-1}$.

In the afterglow spectrum (Fig. 5), the contribution of $\mathrm{FeO}$ "orange arc" emission between 550 and $650 \mathrm{~nm}$ is very likely, as was previously found by Jenniskens et al. (2000) in the afterglow spectrum of a Leonid fireball. In addition, a broadening of lines with respect to the fireball spectrum (Fig. 4) is evident, especially for the $\mathrm{Na} \mathrm{I-1}$ and Mg I-2 multiplets. This effect is mostly related to the slitless nature of the spectrograph and the fact that the volume in the atmosphere of the emitting plasma was larger after the final flare took place. Therefore, this broadening would not be the result of the Doppler effect from thermal motions of ions and free electrons. Since the spectrograph that recorded the afterglow spectrum is based on an 8 bits (256 gray levels) CCD device, the main lines in this signal were saturated at the beginning of the train formation. For this reason, the actual intensity of these lines could not be obtained until after some time. 


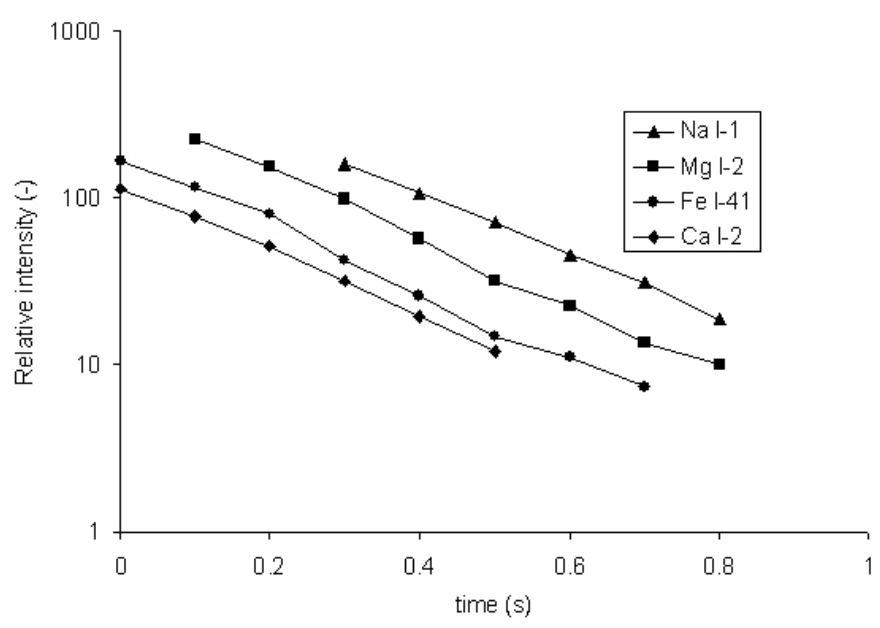

Fig. 7. Evolution with time of the relative intensity of the main emission lines identified in the afterglow spectrum.

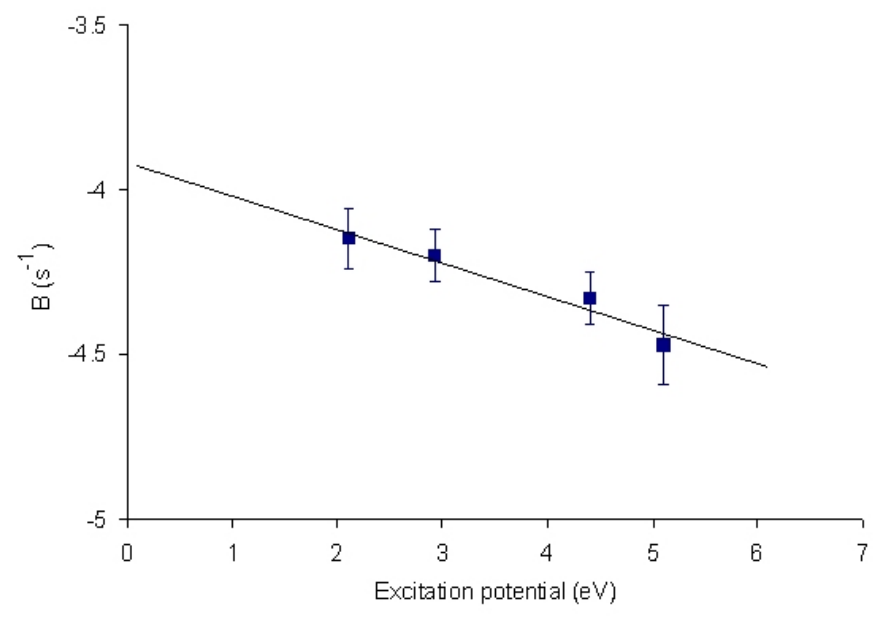

Fig. 8. Dependence of the decay exponent $B$ in Eq. (4) on the excitation potential.

Figure 7 shows the variation with time of the relative intensity (after it was corrected for the instrumental efficiency) of the unsaturated emission lines corresponding to the multiplets $\mathrm{Na} \mathrm{I}-1$ (588.9 nm), Mg I-2 (517.2 nm), Ca I-2 (422.6 nm), and Fe I-41 $(441.5 \mathrm{~nm})$. We found an exponential decrease with time of the intensity of these lines. This behavior was also found for the lines in the afterglow spectrum of Leonid fireballs (Borovička \& Jenniskens 2000). Therefore the relative intensity I of the abovementioned lines was fitted to this equation:

$I=I_{0} \exp (B t)$.

The values obtained for the parameters $I_{0}$ and $B$ in Eq. (4) are summarized in Table 3 for each line appearing in Fig. 7. As this table suggests, the decay rate $B$ depends on the excitation potential $E_{\mathrm{k}}$, with the slowest decays corresponding to the lowest values of $E_{\mathrm{k}}$. This suggests that the observed decrease in intensity during the first $0.8 \mathrm{~s}$ in the afterglow spectrum is mainly controlled by a temperature-driven mechanism in the meteor train (Borovička \& Jenniskens 2000). The dependence of $B$ on $E_{\mathrm{k}}$ for the multiplets listed in Table 3 can be clearly seen in Fig. 8. Within the experimental uncertainty, this dependence can
Table 4. Calculated values of the parameters in Eq. (4) for the main multiplets identified in the afterglow spectrum.

\begin{tabular}{lcccc}
\hline \hline Multiplet & $\lambda(\mathrm{nm})$ & $E_{\mathrm{k}}(\mathrm{eV})$ & $I_{0}$ (a.u.) & $B\left(\mathrm{~s}^{-1}\right)$ \\
\hline Ca I-2 & 422.6 & 2.93 & 113.5 & $-4.20 \pm 0.09$ \\
Fe I-41 & 441.5 & 4.41 & 169.4 & $-4.33 \pm 0.08$ \\
Mg I-2 & 517.2 & 5.10 & 355.6 & $-4.47 \pm 0.12$ \\
Na I-1 & 588.9 & 2.10 & 559.8 & $-4.15 \pm 0.08$ \\
\hline
\end{tabular}

Notes. The excitation potential of the upper level $\left(E_{\mathrm{k}}\right)$ is also indicated.

be considered to be linear, as the continuous line in this plot shows. Therefore we fitted the values of the decay exponent $B$ to the following equation:

$B=B_{0}+D E_{\mathrm{k}}$

where the parameters $B_{0}$ and $D$ yield $-3.91 \mathrm{~s}^{-1}$ and $-0.10 \mathrm{~s}^{-1} \mathrm{eV}^{-1}$, respectively. A similar behaviour was found by Borovička \& Jenniskens (2000) from the analysis of a mag. -13 Leonid fireball. Thus, the afterglow spectrum of this bolide, which was produced by a $1 \mathrm{~kg}$ meteoroid with an initial (preatmospheric) velocity of $72 \mathrm{~km} \mathrm{~s}^{-1}$, also evolved according to a temperature driven mechanism with $D=-1.5 \mathrm{~s}^{-1} \mathrm{eV}^{-1}$.

\section{Conclusions}

We have studied a bright fireball observed over Spain on April 13, 2013. From the analysis of this event, which was named Serranillos del Valle, we found that the parent meteoroid had an initial mass of $40 \pm 5 \mathrm{~kg}$ and penetrated the atmosphere to a final height of $68.1 \pm 0.5$ above the ground level. The survival of meteorites was discarded. The event exhibited several flares along its trajectory. The maximum luminosity of the bolide, corresponding to an absolute magnitude of $-13.0 \pm 0.5$, occurred during the first of these flares. The calculated orbit, atmospheric penetration, and tensile strength of the meteoroid are consistent with a cometary origin for this particle. In particular, the orbital elements reveal that the meteoroid had a sporadic origin and was following a JFC orbit before impacting the Earth. No potential parent comet was found among the objects currently listed in the NeoDys and Minor Planet Center databases, however, which points to the existence of a relatively high-inclination JFC that has not yet been discovered. The results also indicate that the meteoroid was very fragile, with a bulk density of about $0.3 \mathrm{~g} \mathrm{~cm}^{-3}$.

The analysis of the emission spectrum produced by the fireball revealed the chondritic nature of the progenitor meteoroid. In addition, several emission lines produced by $\mathrm{Na}, \mathrm{Mg}, \mathrm{Ca}$, and Fe were identified in the afterglow spectrum. The brightness of these lines decreased exponentially with time. Our results reveal that this decrease was controlled by a temperature-driven mechanism.

Acknowledgements. Meteor stations at Sevilla, La Hita, Huelva and El Arenosillo have been funded by the first author. We acknowledge support from the Spanish Ministry of Science and Innovation (Projects AYA2009-13227 and AYA2011-26522). We also thank the AstroHita Foundation for its support in the establishment and operation of the automated meteor observing station located at La Hita Astronomical Observatory (La Puebla de Almoradiel, Toledo, Spain).

\section{References}

Abe, S., Ebizuka, N., Murayama, H., et al. 2005, Earth Moon Planets, 95, 265 Babadzhanov, P. B., \& Kokhirova, G. I. 2007, Adv. Space Res. 39, 533 
A\&A 569, A104 (2014)

Borovička, J. 1993, A\&A, 279, 627

Borovička, J. 1994, Planet. Space Sci., 42, 145

Borovička, J. 2005, Earth Moon Planets 97, 279

Borovička, J. 2006, J. RAS Canada, 100, 194

Borovička, J., \& Jenniskens, P. 2000, Earth Moon Planets, 8283, 399

Borovička, J., Koten, P., Spurny, P., Boček, J., \& Stork, R. 2005, Icarus, 174, 15

Bronshten, V. A. 1981, Geophysics and Astrophysics Monographs (Dordrecht: Reidel)

Ceplecha, Z. 1987, Bull. Astron. Inst. Cz., 38, 222

Ceplecha, Z., \& McCrosky, R. E. 1976, J. Geophys. Res., 81, 6257

Drummond, J. D. 1981, Icarus, 45, 545

Jenniskens, P. 2004, Adv. Space Res., 33, 1444

Jenniskens, P. 2007, Adv. Space Res., 39, 491

Jenniskens, P. 2008, Icarus, 194, 13

Jenniskens, P., Lacey, M., Allan, B. J., Self, D. E., \& Plane, J. M. C. 2000, Earth Moon Planets, 82, 429

Jopek, T. J. 1993, Icarus 106, 603

Koten, P., Borovička, J., Spurny, P., et al. 2006, MNRAS, 366, 1367

Madiedo, J. M. 2014, Earth Planets \& Space, 66, 70

Madiedo, J. M., \& Trigo-Rodríguez, J. M. 2008, Earth Moon Planets, 102, 133

Madiedo, J. M., Trigo-Rodríguez, J. M., Ortiz, J. L., \& Morales, N. 2010 Advances in Astronomy 2010, 1
Madiedo, J. M., Trigo-Rodríguez, J. M., \& Lyytinen, E. 2011, NASA/CP-2011216469, 330

Madiedo, J. M., et al. 2013a, 44th Lunar and Planetary Science Conf., LPI Contribution No. 1719, 1116

Madiedo, J. M., Trigo-Rodríguez, J. M., Williams, I. P., Ortiz, J. L., \& Cabrera, J. 2013b, MNRAS, 431, 2464

Madiedo, J. M., Trigo-Rodríguez, J. M., Lyytinen, E., et al. 2013c, MNRAS, 431, 1678

Madiedo, J. M., Trigo-Rodríguez, J. M., Konovalova, N., et al. 2013d, MNRAS, 433, 571

Madiedo, J. M., Trigo-Rodríguez, J. M., Ortiz, J. L., Castro-Tirado, A. J., Cabrera-Caño J. 2014, Icarus, in press

Milley, E. P., Hawkes, R. L., \& Ehrman, J. M. 2007, MNRAS, 382, L67

Moore, C. E. 1945, in A Multiplet Table of Astrophysical Interest (Princeton NJ: Princeton University Observatory), Contribution No. 20

Southworth, R. B., \& Hawkins, G. S. 1963, Smithson Contr. Astrophys., 7, 261

Trigo-Rodríguez, J. M., \& Llorca, J. 2006, MNRAS, 372, 655

Trigo-Rodríguez, J. M., \& Llorca, J. 2007, MNRAS, 375, 415

Trigo-Rodríguez, J. M., Madiedo, J. M., Williams I. P., et al. 2009, MNRAS, 394,569

U.S. Standard Atmosphere 1976, NOA-NASA-USAF, Washington

Valsecchi, G., Jopek, T., \& Froeschlé C. 1999, MNRAS, 304, 743 